\title{
Imaging for Epilepsy Surgery
}

\author{
Vamsidhar Chavakula, MD ${ }^{1}$ G. Rees Cosgrove, MD, FRCSC ${ }^{1}$
}

1 Department of Neurosurgery, Brigham and Women's Hospital, Harvard Medical School, Boston, Massachusetts

Semin Neurol 2017;37:580-588.
Address for correspondence Vamsidhar Chavakula, MD, Department of Neurosurgery, Brigham and Women's Hospital, Harvard Medical School, Boston, MA 02115-6195 (e-mail: vchavakula@partners.org).

\begin{abstract}
Keywords

- epilepsy

- MRI

- PET

- EEG

- surgery

Successful epilepsy surgery requires accurate localization of the zone of seizure onset and its complete removal without causing any permanent neurological deficits. While clinical semiology, ictal EEG recordings, and neuropsychological testing are all useful in defining the seizure focus, advanced neuroimaging has emerged as the most important localizing tool. Structural and metabolic imaging can now identify subtle cortical abnormalities that if consistent with other presurgical evaluation investigations, can improve surgical outcomes. Functional imaging can also be helpful in defining eloquent cortex and its relationship to planned surgical resection sites to reduce the risk of neurological impairment. This article explores several advanced neuroimaging techniques and their role in the surgical treatment of epilepsy.
\end{abstract}

Approximately 20 to $30 \%$ of patients with epilepsy are considered drug refractory, ${ }^{1}$ defined by the International League Against Epilepsy (ILAE) as the persistence of seizures despite adequate trials of two well-tolerated and appropriately chosen anti-epileptic drugs (AEDs) under the supervision of an experienced neurologist over the course of at least 1 year. ${ }^{2}$ This population represents the potential pool of candidates for surgical treatment, but not all of these patients are amenable to resective surgery. Successful surgical intervention for epilepsy requires a thorough investigation to identify those patients with clearly localizable epileptic foci, with the goal being freedom from seizure with no permanent neurologic deficits.

The goal of any presurgical evaluation in a patient with drug-resistant epilepsy is to identify a localized region of epileptic cortex and subsequently resect or disconnect that abnormal cortex. The initial investigation relies on a detailed clinical history and neurologic exam with consultation of both the patient and family members who have witnessed the seizures of the patient. The presence of auras, semiology, and timing of seizures are vital pieces of knowledge that inform seizure onset localization. Signs and symptoms, suggestive of a focal seizure onset, are further investigated through long-term video electroencephalogram (EEG) monitoring. Additional testing may include behavioral evaluation and neuropsychological testing. Various neuroimaging tech- niques are also employed to identify structural and/or metabolic abnormalities that may be the source of the seizures. ${ }^{3}$ In the perfect scenario, all data streams are concordant with ictal EEG, confirming that the clinical seizures do indeed arise from a clearly identified cortical region. However, in many cases, there remains some level of discordance in the functional and anatomic seizure localizations, and in these situations, invasive intracranial investigation with implanted electrodes may also be necessary to determine the precise zone of seizure onset.

Most focal epilepsy arises in the temporal lobe, notably the mesial temporal lobe, followed by the frontal, parietal, and occipital lobes. ${ }^{4}$ In some cases, the epileptic zone is multilobar or even panhemispheric. In cases of clear cut mesial temporal sclerosis (MTS), between 65 and $85 \%$ of patients are cured of epilepsy following resection. ${ }^{5,6}$ Surgical success is much less predictable when bilateral MTS is present or when no identifiable temporal lobe lesion exists. This is even true in the extratemporal epilepsies. The most important predictor of a favorable surgical outcome is the identification of a clear cut structural lesion that is consistent with the clinical semiology of the seizures and the ictal EEG onset. ${ }^{7}$ Therefore, advanced neuroimaging techniques have become increasingly important in identifying and defining epileptogenic lesions that might previously have gone undetected.
Issue Theme Neuroimaging; Guest Editor, Joshua P. Klein, MD, PhD, FANA, FASN, FAAN
Copyright $\odot 2017$ by Thieme Medical Publishers, Inc., 333 Seventh Avenue, New York, NY 10001, USA. Tel: +1(212) 584-4662.
DOI https://doi.org/ 10.1055/s-0037-1607986. ISSN 0271-8235. 
In this article, we discuss the advances in neuroimaging techniques that have allowed surgeons to more precisely identify the epileptic zone and subtle lesions that may not have been detectable in the past.

\section{Magnetic Resonance Imaging}

Magnetic resonance imaging (MRI) is the most useful imaging modality employed in the investigation of epilepsy. The most common sequences to obtain are thin cut T1- and T2-weighted images to evaluate white and gray matter distinction as well as evaluate the fluid spaces. Fluid attenuated inversion recovery (FLAIR) sequences allow for the evaluation of edema, subcortical sclerosis, and focal atrophy. Hemosiderin and calcium-sensitive sequences, such as gradient echo (GRE) and susceptibilityweighted imaging (SWI), can also be useful to detect small vascular abnormalities or evidence of remote hemorrhage. Most images are acquired at $1.5 \mathrm{~T}$ in the standard axial, coronal, and sagittal planes, though oblique coronal slices perpendicular to the long axis of the hippocampus are useful in suspected cases of MTS. This particular slice orientation can allow for better comparison of hippocampi to determine the degree of asymmetry between the two sides as well as any loss of internal architecture (-Fig. 1).

Careful examination of the images is the responsibility of the epilepsy surgery team, since every presurgical test informs another, and all investigations are complementary. Seizure semiology and scalp EEG often suggest where the zone of seizure onset is most likely and focus attention to that area or lobe of interest. If a lesion is suspected, then additional images can be obtained in coronal or oblique slices through the area of interest. This can often convert the idiopathic nonlesional case into a lesional case with a more favorable surgical outcome.

\section{High-Magnetic Field Strength Imaging}

One of the most touted advances in MRI has been the development of higher magnetic field strength. Initially, small surface coils were placed over the area of interest to improve spatial resolution, but these could only improve visualization under the coil, and therefore placement was extremely important (-Fig. 2). The advantages of 3T MRI and higher magnetic field imaging are improved spatial and contrast resolution over the entire brain, with the detractions being increased susceptibility to motion artifact and increased imaging times. The MR image clarity at 3T can certainly improve the level of confidence that a lesion has been identified, but in most cases, all these lesions can also be seen at $1.5 \mathrm{~T}$, at least in retrospect. $^{8}$ The difference is in the certainty of the diagnosis, which is very important in making a surgical decision (-Fig. 3).

While 3T MRI machines are now widely available, an increasing number of $7 \mathrm{~T}$ machines are currently being deployed. In one prospective study, 21 consecutive patients, with evidence of focal onset seizures but lacking an identifiable lesion on conventional MRI, underwent further imaging using 7T MRI. In 29\% of these patients, GRE and FLAIR, performed at 7T strength, revealed a distinct lesion, with histopathological diagnosis of focal cortical dysplasia in all patients who underwent surgical resection. ${ }^{9}$ The same group also reported that $7 \mathrm{~T}$ imaging allowed for more effective visualization of foci of polymicrogyria. ${ }^{10}$

\section{Volumetric Analysis}

High-resolution thin cut MRI allows for the construction of advanced volumetric and morphological models. Volumetric analysis of the hippocampus has been a tool in the evaluation of temporal lobe epilepsy (TLE), with seizure lateralization occurring primarily on the side with reduced hippocampal volume. ${ }^{11}$ These quantitative methods are useful in detecting subtle mesial temporal atrophy.

Methods are also available to render these three-dimensional (3D) volumetric MRI datasets into accurate cortical surface renderings of the individual patient's brain. Cortical anatomy can be examined for subtle abnormalities of gyral and sulcal patterns. These 3D images can also be used to visualize the location and extent of subcortical abnormalities because the underlying gray matter and white matter may be

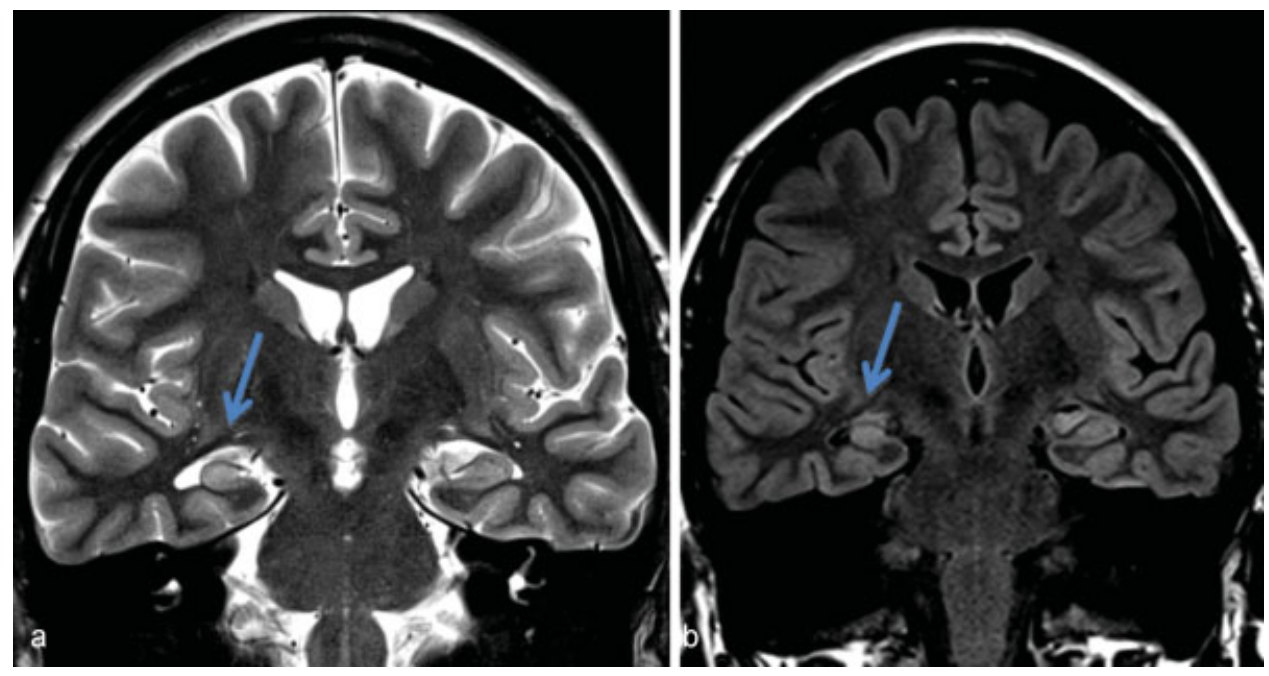

Fig. 1 Oblique coronal T2 (a) and T2-FLAIR (b) MR images in a patient with intractable complex partial seizures and a history of febrile convulsions as an infant. Note the slightly decreased volume and loss of internal architecture on T2-weighted images as well as the increased $\mathrm{T} 2$ signal intensity in the right hippocampus (arrows). FLAIR, fluid attenuation inversion recovery; MR, magnetic resonance. 

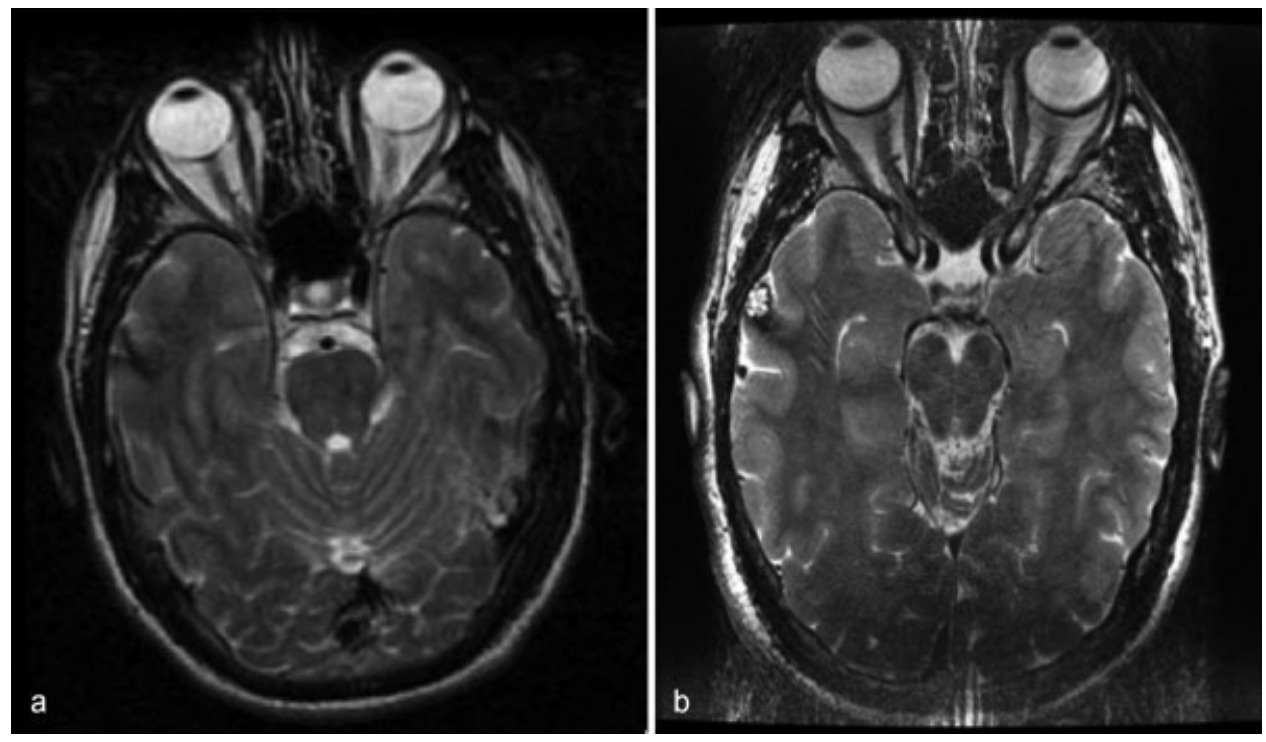

Fig. 2 Standard T2 fast spin echo axial MR image (a) and image obtained with a surface coil over the temporal regions (b) in a patient with intractable complex partial seizures and a very active interictal spike discharge on scalp EEG in the right temporal leads. The abnormality was clearly identified as a small cavernous angioma, and in retrospect, there was a suspicious area on standard MRI, but this was not clear enough on its own to make a firm diagnosis. EEG, electroencephalogram; MR, magnetic resonance.
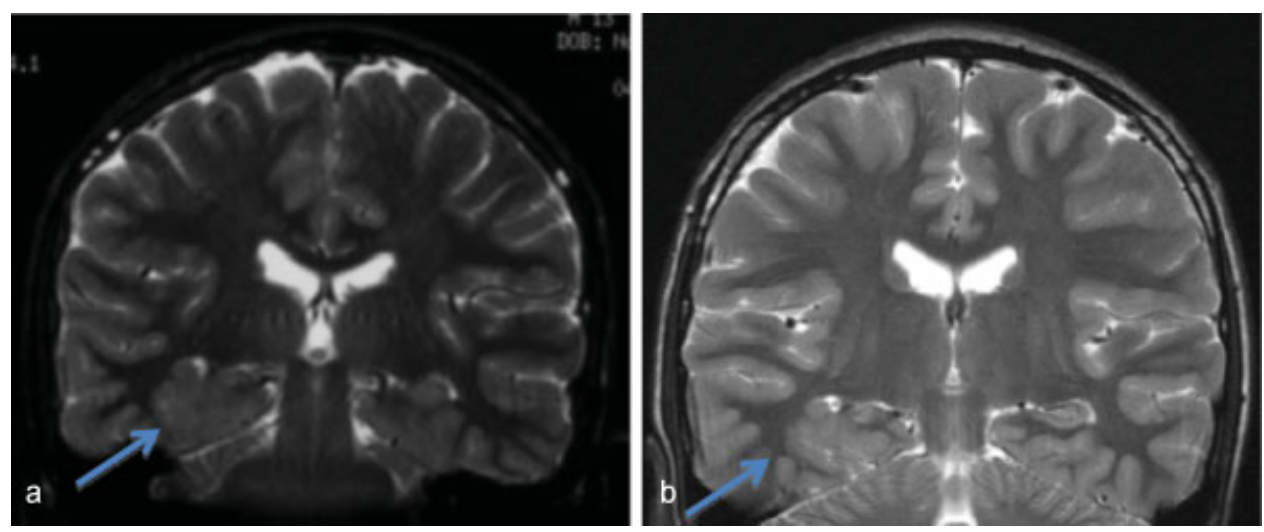

Fig. 3 Coronal T2-weighted MR images at 1.5T (a) and 3.0T (b) in a young boy with intractable seizures. The initial MRI at 1.5T was interpreted as normal, but the dysplastic cortex along the collateral sulcus and the periventricular gray matter in the right temporal lobe that is so evident on the $3 \mathrm{~T}$ images can also be appreciated in retrospect on the 1.5T images (arrows). MR, magnetic resonance; MRI, magnetic resonance imaging.

examined using readily available computerized image processing tools ${ }^{12}$ (-Fig. 4).

Advanced image processing techniques can also create cortical thickness maps with automated color-coded measurements of the cortical mantle and the gray-white junction. The human cortical gray matter is typically 2 to $4 \mathrm{~mm}$ thick, but varies between Brodmann's areas. Cortical dysplasias are often characterized by thickened and disorganized gray matter, and subtler cases, too difficult to see on standard imaging, can sometimes be detected with these techniques. ${ }^{13,14}$ At the very least, areas that appear thickened on cortical thickness maps can be examined much more carefully and attentively on the original MR image sequences. Quantitative imaging can also define the total volumes of gray and white matter in each lobe, which may also inform the trained observer that a subtle lobar abnormality exists that could not be appreciated on direct inspection. ${ }^{15}$ Qualitative evaluation is currently in clinical use, while quantitative automated methods remain under investigation. $^{16,17}$

\section{Myelin Maps}

An even newer technique for measuring myelin concentration using multicomponent-driven equilibrium single-pulse observation of $\mathrm{T} 1$ and $\mathrm{T} 2$ (McDESPOT) may also play an important role in evaluating the white matter of patients with epilepsy, especially pediatric patients. ${ }^{18}$ This technique compares the myelin concentration of the subject with an age-matched control database and highlights differences. These differences are then presented visually for examination and can be used to focus attention on the cortical area of interest ( - Fig. 5).

\section{Diffusion Tensor Imaging}

Diffusion tensor imaging (DTI) is a relatively new technique that can better visualize white matter. The directionality of 

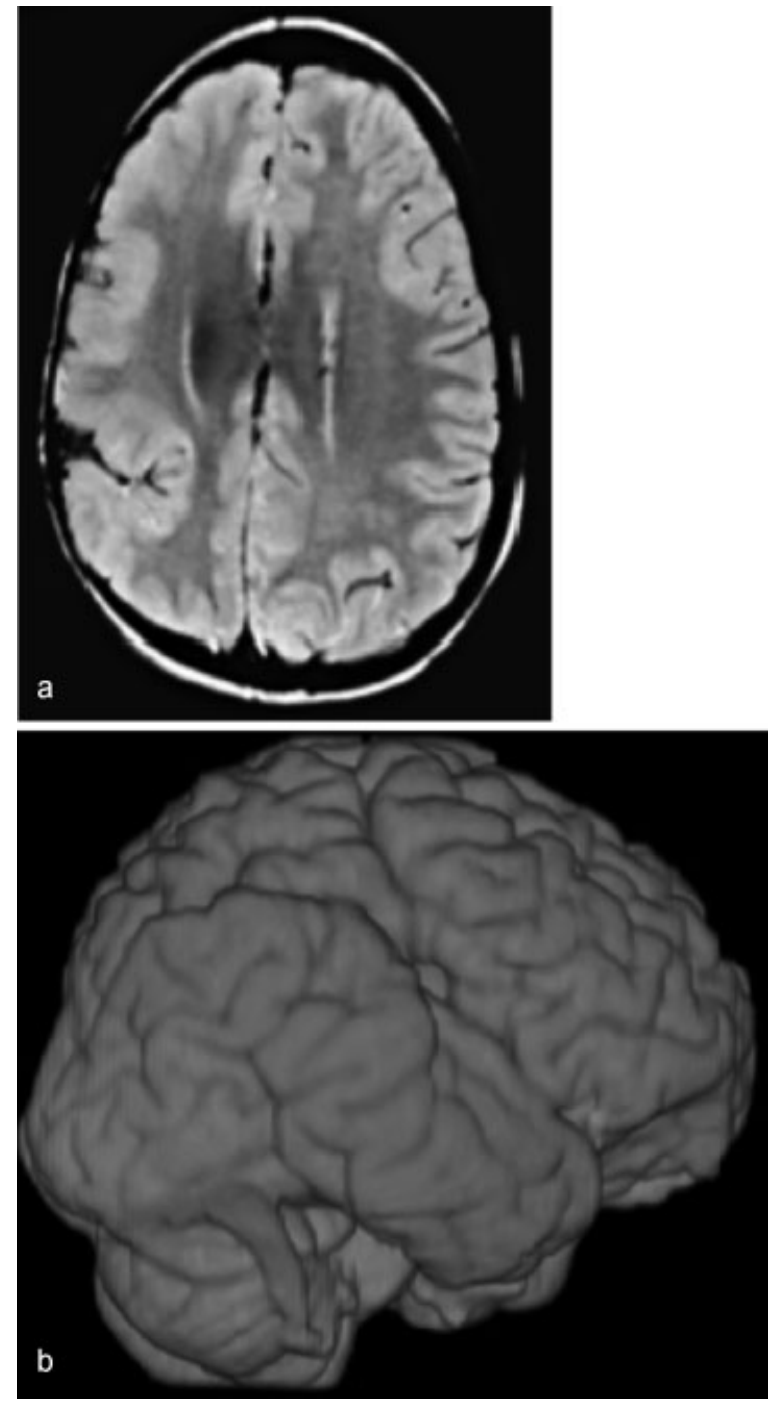

Fig. 4 Axial T2-weighted MRI (a) of a child with intractable generalized seizures due to right hemispheric schizencephaly and a 3D surface rendering (b) of the same child that demonstrates the extent of the perisylvian gyral abnormalities more clearly. Note the deepened sylvian fissure that extends into the parietal lobe; the wide flattened gyri; and the lack of normal tertiary sulcal patterns. MRI, magnetic resonance imaging.

the white matter fiber tracts is color coded based upon the differential mobility of water molecules along versus perpendicular to the fiber bundles, a term known as anisotropy. Where axonal bundles are intact and parallel, anisotropy is high; where axonal tracts are spatially disorganized or intermingled, anisotropy is low. ${ }^{13,19-21}$ These color-coded anisotropy maps can be analyzed to see if the coefficient of anisotropy is maintained or normal in the various lobes of the brain as an indirect measure of dysfunctional connections. In cases of medial temporal epilepsy, it has been found that associated limbic white matter tracts, such as the fornix and cingulum, can show evidence of injury. ${ }^{22}$ The use of this technique in epilepsy surgery remains investigational.

Diffusion tensor imaging has become a unique tool in surgical planning by identifying eloquent tracts, such as the corticospinal tract for motor function or the arcuate fasciculus for language. ${ }^{23}$ For deep epileptic foci near eloquent pathways, tractography may be used to generate a surgical plan that avoids injury. ${ }^{24}$ In MTS, tractography is being used to evaluate Meyer's loop, which carries contralateral superior visual quadrant information. Following either anterior temporal lobectomy or selective amygdalohippocampectomy, greater than $40 \%$ of patients experience visual field deficits due to disruption in Meyer's loop. ${ }^{25}$ In one series of 12 patients, selective amygdalohippocampectomy was performed either through the subtemporal approach or a transcortical approach, depending on which method allowed for avoidance of Meyer's loop as visualized by DTI tractography. Postoperatively, only $25 \%$ of patients experienced a visual field deficit. $^{26}$

A future direction for DTI application is the development of functional connectome maps for the elucidation of functional seizure tracts. In these methods, DTI maps are used to develop a topographical map of seizure network nodes. Through computational simulations of seizure spread, the most important nodes or foci for initiation and propagation of seizures may be identified, and targeted resection may be performed. This remains a purely research endeavor at the moment and not ready for clinical application. ${ }^{27}$

From a mathematical viewpoint, DTI is limited by the fact that each 2-mm voxel is treated as a single diffusion compartment (white matter, gray matter, or CSF only), whereas anatomically, such a voxel would actually comprise some combination of white matter, gray matter, and $\mathrm{CSF}^{28}$ Two other analyses being investigated include Composite Hindered and Restricted Model of Diffusion (CHARMED) and Neurite Orientation Dispersion and Density Imaging (NODDI). In CHARMED, during reconstruction, each voxel is modeled with an intracellular and an extracellular component. By removing the artifact from the extracellular component, one anticipates higher resolution of axonal integrity and direction. ${ }^{29}$

NODDI models each voxel as a combination of three compartments: intracellular, extracellular, and CSF. Measures that may be obtained through this analysis include the orientation dispersion index (ODI), which reflects the degree to which neurites are dispersed. It also allows for the calculation of the intracellular volume fraction, which is a reflection of neurite density. ${ }^{30}$ In one preliminary study, it has been shown that NODDI can be used to identify occult focal cortical dysplasia. ${ }^{31}$

\section{Perfusion Imaging}

Although not in widespread use, a variety of MRI sequences have been developed with application for imaging in epilepsy. Arterial spin labeling (ASL) is a contrast-free perfusion measuring sequence and involves radiofrequency bursts in the neck to cause inverted magnetization of arterial water. Following a delay to allow the inverted water to perfuse the cortex, the desired regions are imaged. Control labeling is also performed in which the magnetic inversion step is not performed. By comparing the differences in magnetic inversion between the ASL and control sequences, one can obtain an estimate of cerebral perfusion at various locations. Limitations of ASL stem from a relatively low signal to noise ratio, 


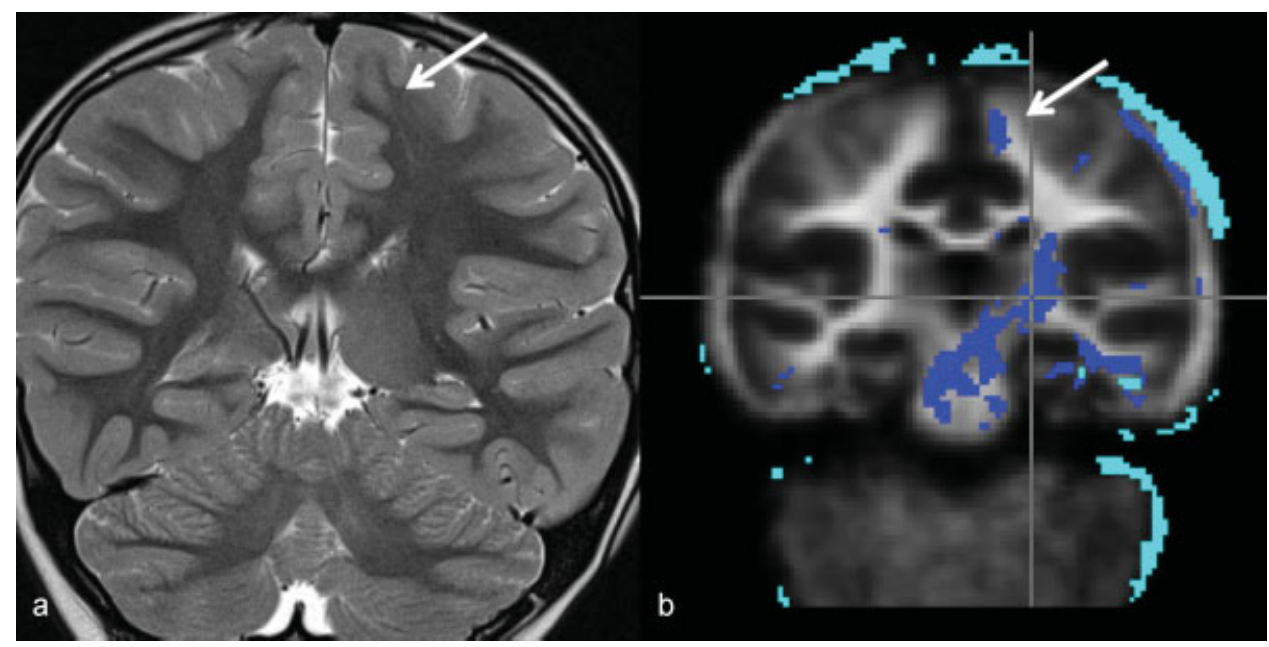

Fig. 5 T2-weighted coronal images of a 6-year-old girl with severe intractable seizures with a left supplementary motor area focus. All MRIs were reportedly normal, but a small transmantle dysplasia was identified in the left superior frontal gyrus (a, arrow) and McDESPOT imaging demonstrated a focal area of decreased myelin concentration in the same area (b, arrow) along with more diffuse myelin loss in the deeper white matter tracts. McDESPOT, multicomponent-driven equilibrium single-pulse observation of T1 and T2; MRI, magnetic resonance imaging.

which is susceptible to artifacts. ${ }^{32}$ In one study of 16 patients with epilepsy, comparing ASL to PET, despite having slightly decreased resolution, ASL was able to identify the same abnormalities in perfusion as PET imaging. ${ }^{33}$ In a more recent study of 164 patients with seizures, ASL imaging was able to detect alterations in perfusion in $39 \%$ of patients, with highest sensitivity if the imaging was performed within 5 hours of seizure onset. ${ }^{34}$ Another perfusion-weighted sequence is dynamic susceptibility contrast (DSC) MR perfusion. In this modality, alterations in $\mathrm{T} 2$ signal are induced by passage of paramagnetic contrast agents through the cerebral microvasculature, which can then be used to determine the perfusion of tissue with measures including cerebral blood volume (CBV) and cerebral blood flow (CBF). ${ }^{35}$ DSC imaging may be distorted or inaccurate in regions of calcification or hemorrhage due to its variable effects on the contrast agent signal. Despite the differences in techniques, both ASL and DSC have been used to lateralize temporal lobe epilepsy without the need for radioactive agents. ${ }^{36}$

\section{Functional MRI}

Functional MRI (fMRI) is based on the principle that metabolically active regions of brain have increased blood flow, known as activation flow coupling. The $\mathrm{T} 2{ }^{*}$-weighted blood oxygenation level dependent (BOLD) signal is viewed as a reflection of neural activity. ${ }^{37}$ During an fMRI study, the patient is asked to perform or imagine a series of tasks while actively being imaged. Extremity movement tasks allow for the detection of sensorimotor regions of brain, while speech and comprehension tasks allow for identification of regions responsible for language.

The utility of fMRI in epilepsy surgery relates to the identification of eloquent regions of brain; notably motor and sensory function when lesions are adjacent to these cortical areas. Pending the location of the lesion, surgical approaches as well as extent of resection may be decided. Memory function may also be assessed via fMRI, but this method remains in development and does not obviate the need for invasive WADA testing when indicated. The more common use of fMRI is for lateralization and localization of language (-Fig. 6). In cases of medial temporal epilepsy, the side of language dominance determines the extent of posterior resection. On the nondominant hemisphere, up to $6 \mathrm{~cm}$ of temporal lobe may be resected, while on the dominant side, up to $4 \mathrm{~cm}$ may be safely resected.

Some investigation has been done into simultaneous scalp EEG/fMRI as a method for coupling the temporal resolution of EEG with the spatial resolution of fMRI. The promise of such imaging is the ability to follow the propagation of the seizure in real time across the brain and thereby identify the entire epileptic network. ${ }^{38}$ Although actively capturing an ictal event is quite difficult, it is hoped that interictal spikes may also be used to mark subthreshold events.

\section{Magnetoencephalography}

As a technology, magnetoencephalography (MEG) is most similar in functionality to EEG. The premise of MEG is that synchronous neural activity leads to the generation of magnetic dipoles, which are recorded by a multidetector array (-Fig. 7). In epilepsy, MEG has been investigated to isolate the onset and propagation of interictal spikes as a proxy for epileptogenic activity; however, full clinical utility has not been achieved. ${ }^{39}$ Benefits of MEG are that magnetic dipoles do not experience artifacts from skull and muscle artifacts and so allow for noninvasive recording simultaneously over the entire brain. Limitations stem from the low signal to noise ratio that exists if not enough concurrent dipoles are present. Also, given the expense of imaging, long-term MEG is often not possible. 

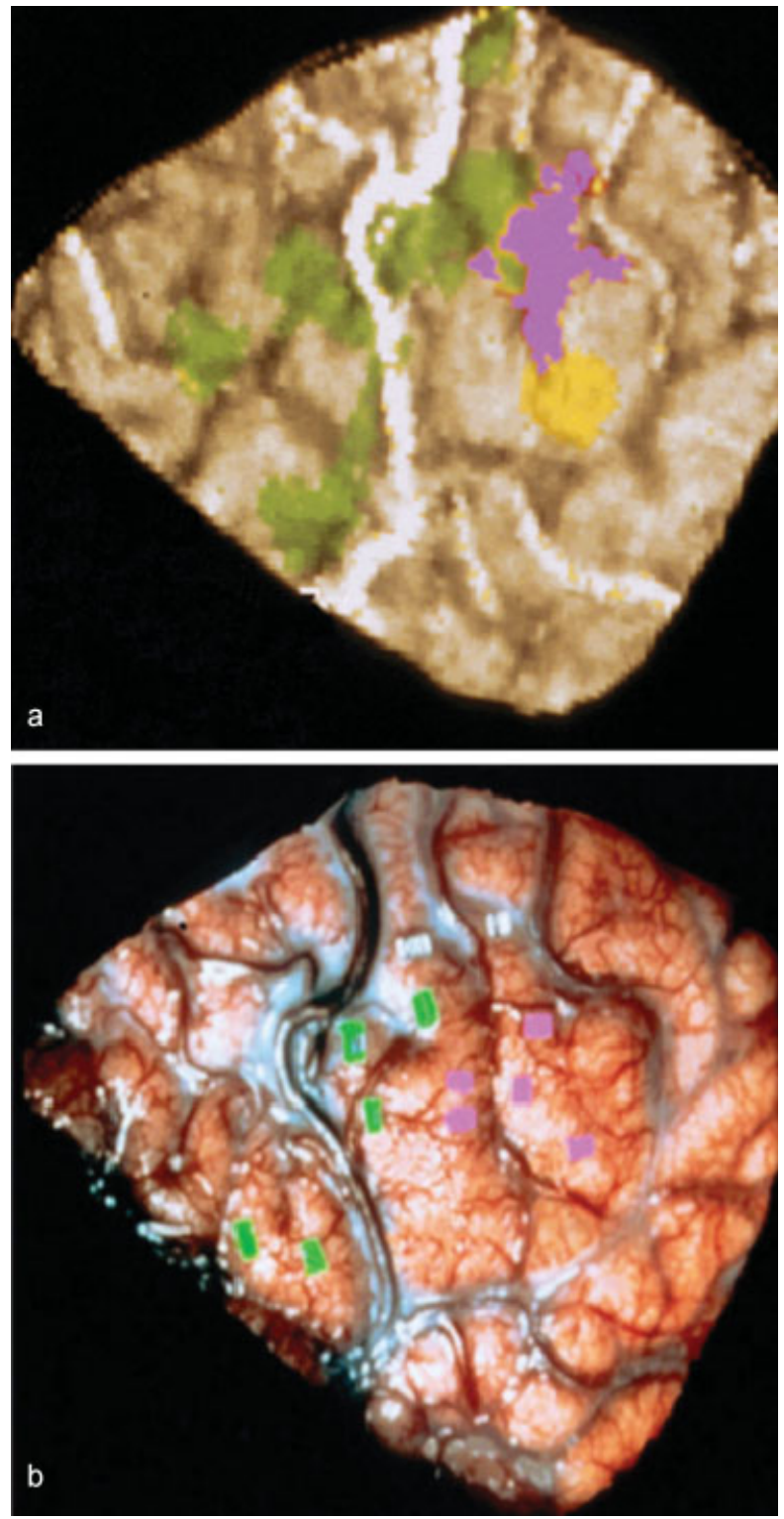

Fig. 6 Functional MRI of a 26-year-old man with new onset seizures characterized by ictal coughing and speech arrest. MRI demonstrated a small enhancing lesion in the left subcentral gyrus (a, yellow). The patient underwent fMRI for tongue sensorimotor mapping (mauve) and language mapping (green), and the images were rendered with superimposed cortical venous structures (a, white). Surgery was performed awake with right tongue contraction and sensory responses marked (mauve) and speech arrest (green) marked with tags on the cortical surface (b). Lesionectomy while testing for speech was performed successfully without neurological deficit. Pathology demonstrated ganglioglioma. fMRI, functional MRI.

\section{Positron Emission Tomography}

Fludeoxyglucose F18 positron emission tomography $\left({ }^{18} \mathrm{~F}-\mathrm{FDG}\right.$ PET) imaging is based on the principle that metabolic activity of cortex is reflected in the rate of uptake of glucose. ${ }^{18} \mathrm{~F}$-FDG is a glucose analogue with an attached positron emitting fluoride atom. Imaging may be performed in either the ictal or interictal period. During ictal imaging, there should be evidence of increased metabolic activity at the seizure onset zone, while in interictal PET, there is often evidence of hypometabolism at the seizure onset zone $\mathrm{C}^{40}(\mathbf{- F i g . ~ 8}$ ). In cases of temporal lobe epilepsy, FDG-PET imaging has been found to have greater than $80 \%$ sensitivity, ${ }^{41,42}$ while in cases of extratemporal epilepsy, sensitivity was less. ${ }^{42} \mathrm{An}$ issue with metabolic imaging is spatial resolution, as the region of abnormal activity is oftentimes larger than the true seizure focus. As such, it remains a poor modality for identifying an exact surgical plan of resection.

\section{Single Photon Emission Computed Tomography}

Single photon emission computed tomography (SPECT) is a nuclear medicine imaging modality that relies on the presence of a radioactive tracer to determine both quantitatively and qualitatively the rates of regional cerebral perfusion. Interictal SPECT is less sensitive in detecting alterations in perfusion, with $44 \%$ sensitivity, and as such has fallen out of favor as a primary imaging modality in epilepsy. One method to increase the sensitivity of SPECT is known as subtraction ictal and interictal SPECT coregistered to MRI (SISCOM) and involves the challenge of obtaining SPECT imaging at the time of seizure onset ( - Fig. 9). To properly perform an ictal SPECT, the tracer must be injected and imaging performed within the first minute of seizure onset to most effectively identify the seizure onset zone. Benefits of this method are that changes in perfusion are directly superimposed on MRI imaging to provide more exact anatomic localization of perfusion abnormalities. However, this is a very difficult technique to perform in any institution, and false localizations can occur if there is a delay in injection. A meta-analysis of this modality has not demonstrated significant clinical utility. $^{43}$

\section{Intraoperative Imaging}

In addition to utilizing multimodal imaging for surgical preplanning, the widespread deployment of neuronavigation systems from multiple companies has allowed surgeons to visualize and confirm abnormal lesions in the intraoperative setting. As software sophistication increases, information from both anatomical and functional imaging studies and tractography can be integrated to compare the real-time operative location with alternative functional and anatomic analyses. Hence, following a good registration, the surgeon can confidently maximize resection of lesional tissue while avoiding injury to eloquent tracts. A technology that is also gaining widespread attention is intraoperative MR imaging (iMRI). Using this technology, the patient may be imaged intraoperatively while still under general anesthesia. The availability of this most updated information allows the surgeon the freedom to perform further resection in a targeted fashion at border zones, which may not be readily apparent by visual inspection. In one institutional study of surgery for patients with medically refractory epilepsy, it was found that $12 \%$ of patients underwent second-look resection following iMRI verification of incomplete resection. ${ }^{44}$ 

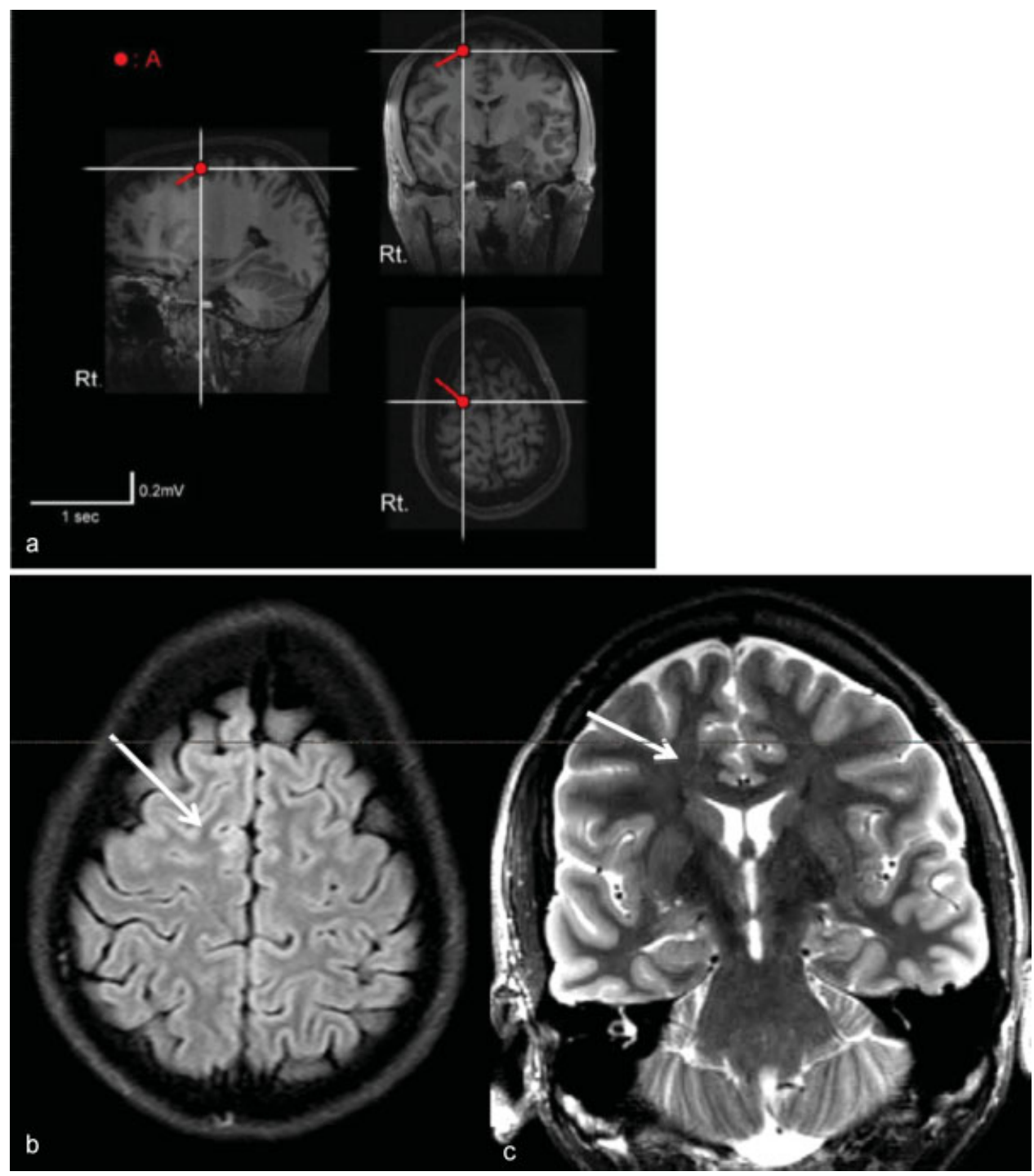

Fig. 7 A 19-year-old male patient had mainly nocturnal seizures beginning with tingling in the left thigh followed by tonic extension of the left arm and flexion of the right arm. Diurnal events were associated with frequent falls. Interictal scalp EEG was unhelpful and ictal EEG appeared bilateral from the onset. MEG was performed and revealed a single dipole source in the right frontal lobe (a, arrows). Reinterpretation of the MRI demonstrated a small area of high-intensity signal in the right parasagittal cortex (b, arrows), and coronal MRI revealed a small transmantle dysplasia (c, arrows). Focal cortical resection of the dysplasia cured the patient of his seizures. Pathology demonstrated a cortical dysplasia Type IIb. EEG, electroencephalogram; MRI, magnetic resonance imaging.

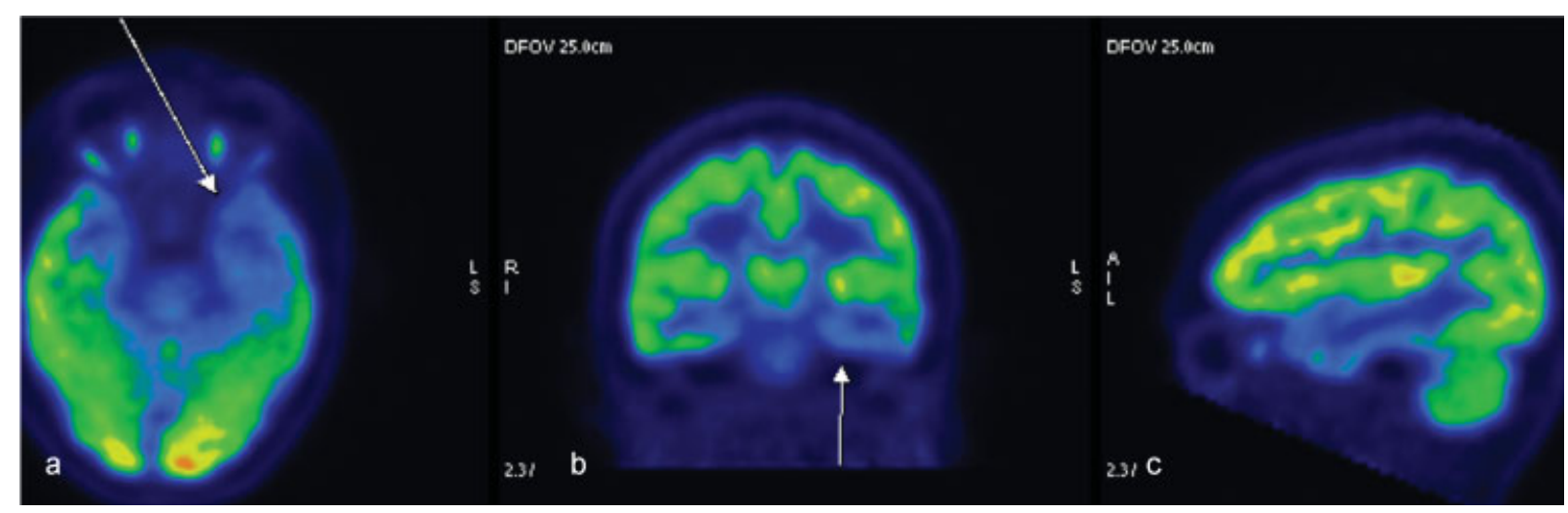

Fig. 8 Interictal PET images in the axial (a), coronal (b), and sagittal (c) planes in an adult with intractable temporal lobe seizures. Note the decreased metabolism in the left temporal lobe affecting primarily the inferior and mesial temporal neocortex ( $\mathbf{a}$ and $\mathbf{b}$ [arrows] and $\mathbf{c}$ ). $\mathrm{PET}$, positron emission tomography. 


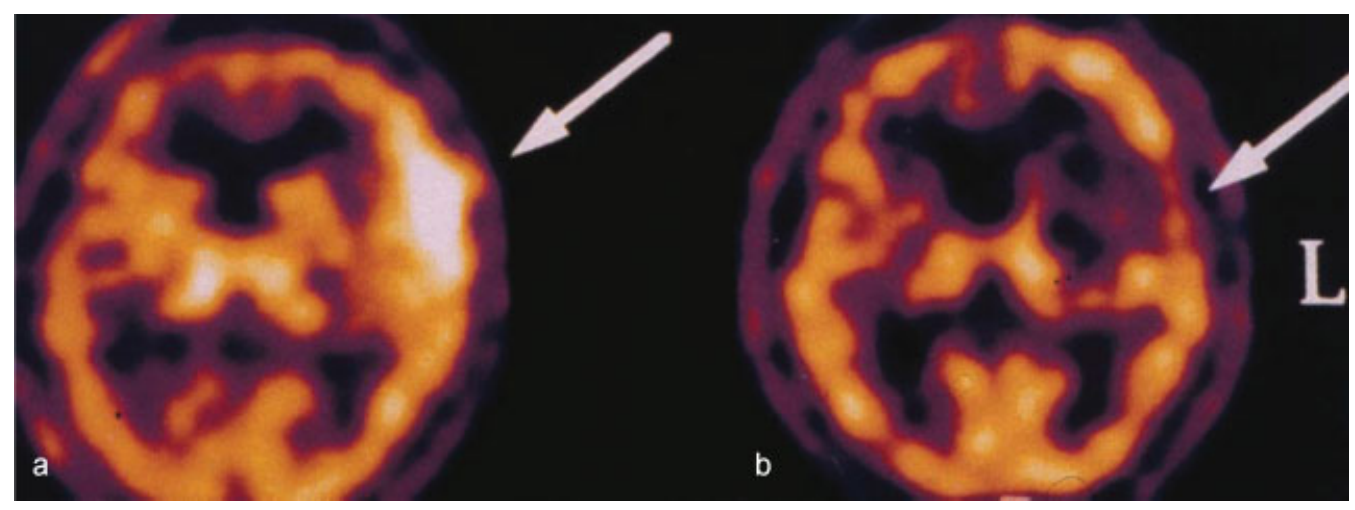

Fig. 9 Ictal and interictal axial SPECT images in a patient with focal and generalized seizures with a suspected frontal or temporal onset. Peri-ictal injection of the radioisotope reveals increased uptake in the left inferior frontal region (a, arrow), confirming a suspicion of focal interictal hypometabolism in the same area (b, arrow). SPECT, single photon emission computed tomography.

\section{Conclusion}

Successful epilepsy surgery relies on the precise localization and subsequent resection of a well-defined epileptogenic focus. A high degree of concordance between multiple data streams yields the highest rates of seizure freedom. By combining information from multiple imaging modalities, the surgeon is more certain of the precise location and extent of the resection target. Advanced imaging techniques are proving valuable at all stages of treatment. At the presurgical stage, novel methods are allowing for the visualization of lesions that previously were undetectable. In cases where standard video EEG may yield equivocal localization, more sensitive imaging modalities allow for the possibility of new targets for invasive recording. Functional imaging and tractography allow for the elucidation of eloquent structures and their avoidance during surgery. With the ability to coregister structural and functional imaging in modern neuronavigation systems, the efficacy and safety of epilepsy surgery are improved.

\section{References}

1 Picot MC, Baldy-Moulinier M, Daurès JP, Dujols P, Crespel A. The prevalence of epilepsy and pharmacoresistant epilepsy in adults: a population-based study in a Western European country. Epilepsia 2008;49(07):1230-1238

2 Kwan P, Arzimanoglou A, Berg AT, et al. Definition of drug resistant epilepsy: consensus proposal by the ad hoc Task Force of the ILAE Commission on Therapeutic Strategies. Epilepsia 2010;51(06): 1069-1077

3 Duncan JS. Imaging in the surgical treatment of epilepsy. Nat Rev Neurol 2010;6(10):537-550

4 Shorvon S. Seizure type and anatomical location of seizures. Handbook of Epilepsy Treatment. Chichester, UK: Wiley-Blackwell; 2010:6-18

5 Jeong SW, Lee SK, Kim KK, Kim H, Kim JY, Chung CK. Prognostic factors in anterior temporal lobe resections for mesial temporal lobe epilepsy: multivariate analysis. Epilepsia 1999;40(12):17351739

6 Wiebe S, Blume WT, Girvin JP, Eliasziw M; Effectiveness and Efficiency of Surgery for Temporal Lobe Epilepsy Study Group. A randomized, controlled trial of surgery for temporal-lobe epilepsy. N Engl J Med 2001;345(05):311-318
7 Berkovic SF, McIntosh AM, Kalnins RM, et al. Preoperative MRI predicts outcome of temporal lobectomy: an actuarial analysis. Neurology 1995;45(07):1358-1363

8 Knake S, Triantafyllou C, Wald LL, et al. 3T phased array MRI improves the presurgical evaluation in focal epilepsies: a prospective study. Neurology 2005;65(07):1026-1031

9 De Ciantis A, Barba C, Tassi L, et al. 7T MRI in focal epilepsy with unrevealing conventional field strength imaging. Epilepsia 2016; 57(03):445-454

10 De Ciantis A, Barkovich AJ, Cosottini M, et al. Ultra-high-field MR imaging in polymicrogyria and epilepsy. Am J Neuroradiol 2015; 36(02):309-316

11 Seidenberg M, Kelly KG, Parrish J, et al. Ipsilateral and contralateral MRI volumetric abnormalities in chronic unilateral temporal lobe epilepsy and their clinical correlates. Epilepsia 2005;46 (03):420-430

12 Xu C, Pham DL, Rettmann ME, Yu DN, Prince JL. Reconstruction of the human cerebral cortex from magnetic resonance images. IEEE Trans Med Imaging 1999;18(06):467-480

13 Bernasconi A, Bernasconi N, Bernhardt BC, Schrader D. Advances in MRI for 'cryptogenic' epilepsies. Nat Rev Neurol 2011;7(02):99-108

14 Hong SJ, Kim H, Schrader D, Bernasconi N, Bernhardt BC, Bernasconi A. Automated detection of cortical dysplasia type II in MRInegative epilepsy. Neurology 2014;83(01):48-55

15 Peng SJ, Harnod T, Tsai JZ, et al. Evaluation of subcortical grey matter abnormalities in patients with MRI-negative cortical epilepsy determined through structural and tensor magnetic resonance imaging. BMC Neurol 2014;14:104

16 Adler S, Wagstyl K, Gunny R, et al. Novel surface features for automated detection of focal cortical dysplasias in paediatric epilepsy. Neuroimage Clin 2016;14:18-27

17 Thesen T, Quinn BT, Carlson C, et al. Detection of epileptogenic cortical malformations with surface-based MRI morphometry. PLoS One 2011;6(02):e16430

18 Spader HS, Ellermeier A, O'Muircheartaigh J, et al. Advances in myelin imaging with potential clinical application to pediatric imaging. Neurosurg Focus 2013;34(04):E9

19 Basser PJ, Pajevic S, Pierpaoli C, Duda J, Aldroubi A. In vivo fiber tractography using DT-MRI data. Magn Reson Med 2000;44(04): 625-632

20 Gross DW. Diffusion tensor imaging in temporal lobe epilepsy. Epilepsia 2011;52(Suppl 4):32-34

21 Sivakanthan S, Neal E, Murtagh R, Vale FL. The evolving utility of diffusion tensor tractography in the surgical management of temporal lobe epilepsy: a review. Acta Neurochir (Wien) 2016; 158(11):2185-2193

22 Concha L, Beaulieu C, Collins DL, Gross DW. White-matter diffusion abnormalities in temporal-lobe epilepsy with and without 
mesial temporal sclerosis. J Neurol Neurosurg Psychiatry 2009;80 (03):312-319

23 Bello L, Gambini A, Castellano A, et al. Motor and language DTI Fiber Tracking combined with intraoperative subcortical mapping for surgical removal of gliomas. Neuroimage 2008;39(01): 369-382

24 Winston GP, Yogarajah M, Symms MR, McEvoy AW, Micallef C, Duncan JS. Diffusion tensor imaging tractography to visualize the relationship of the optic radiation to epileptogenic lesions prior to neurosurgery. Epilepsia 2011;52(08):1430-1438

25 Winston GP. Epilepsy surgery, vision, and driving: what has surgery taught us and could modern imaging reduce the risk of visual deficits? Epilepsia 2013;54(11):1877-1888

26 Thudium MO, Campos AR, Urbach H, Clusmann H. The basal temporal approach for mesial temporal surgery: sparing the Meyer loop with navigated diffusion tensor tractography. Neurosurgery 2010;67(2, Suppl Operative)385-390

27 Hutchings F, Han CE, Keller SS, Weber B, Taylor PN, Kaiser M. Predicting surgery targets in temporal lobe epilepsy through structural connectome based simulations. PLOS Comput Biol 2015;11(12):e1004642

28 Winston GP. The potential role of novel diffusion imaging techniques in the understanding and treatment of epilepsy. Quant Imaging Med Surg 2015;5(02):279-287

29 Assaf Y, Basser PJ. Composite hindered and restricted model of diffusion (CHARMED) MR imaging of the human brain. Neuroimage 2005;27(01):48-58

30 Zhang H, Schneider T, Wheeler-Kingshott CA, Alexander DC. NODDI: practical in vivo neurite orientation dispersion and density imaging of the human brain. Neuroimage 2012;61(04): 1000-1016

31 Winston GP, Micallef C, Symms MR, Alexander DC, Duncan JS, Zhang $\mathrm{H}$. Advanced diffusion imaging sequences could aid assessing patients with focal cortical dysplasia and epilepsy. Epilepsy Res 2014;108(02):336-339
32 Grade M, Hernandez Tamames JA, Pizzini FB, Achten E, Golay X, Smits M. A neuroradiologist's guide to arterial spin labeling MRI in clinical practice. Neuroradiology 2015;57(12):1181-1202

33 Pendse N, Wissmeyer M, Altrichter S, et al. Interictal arterial spinlabeling MRI perfusion in intractable epilepsy. J Neuroradiol 2010;37(01):60-63

34 Yoo RE, Yun TJ, Yoon BW, et al. Identification of cerebral perfusion using arterial spin labeling in patients with seizures in acute settings. PLoS One 2017;12(03):e0173538

35 Essig M, Shiroishi MS, Nguyen TB, et al. Perfusion MRI: the five most frequently asked technical questions. Am J Roentgenol 2013; 200(01):24-34

36 Oner AY, Eryurt B, Ucar M, et al. pASL versus DSC perfusion MRI in lateralizing temporal lobe epilepsy. Acta Radiol 2015;56(04):477-481

37 Kesavadas C, Thomas B. Clinical applications of functional MRI in epilepsy. Indian J Radiol Imaging 2008;18(03):210-217

38 van Graan LA, Lemieux L, Chaudhary UJ. Methods and utility of EEGfMRI in epilepsy. Quant Imaging Med Surg 2015;5(02):300-312

39 Oishi M, Otsubo H, Kameyama S, et al. Epileptic spikes: magnetoencephalography versus simultaneous electrocorticography. Epilepsia 2002;43(11):1390-1395

40 Sarikaya I. PET studies in epilepsy. Am J Nucl Med Mol Imaging 2015;5(05):416-430

41 Garibotto V, Picard F. Nuclear medicine imaging in epilepsy. Epileptologie 2013;30:109-121

42 Won HJ, Chang KH, Cheon JE, et al. Comparison of MR imaging with PET and ictal SPECT in 118 patients with intractable epilepsy. Am J Neuroradiol 1999;20(04):593-599

43 Chen T, Guo L. The role of SISCOM in preoperative evaluation for patients with epilepsy surgery: a meta-analysis. Seizure 2016; 41:43-50

44 Roessler K, Hofmann A, Sommer B, et al. Resective surgery for medically refractory epilepsy using intraoperative MRI and functional neuronavigation: the Erlangen experience of 415 patients. Neurosurg Focus 2016;40(03):E15 